\title{
ON THE BOUNDEDNESS OF DUNKL-TYPE MAXIMAL COMMUTATORS IN THE DUNKL-TYPE MODIFIED MORREY SPACES
}

\author{
Samira A. Hasanli \\ Nakhcivan State University \\ Nakhchivan City, AZ 7012, AZERBAIJAN
}

\begin{abstract}
In this paper we consider the generalized shift operator, associated with the Dunkl operator and we investigate maximal commutators, commutators of singular integral operators and commutators of the fractional integral operators associated with the generalized shift operator.

The boundedness of the Dunkl-type maximal commutator $M_{b, \alpha}$ from the Dunkl-type modified Morrey space $\widetilde{\mathcal{M}}_{p, \lambda, \alpha}(R)$ to $\widetilde{\mathcal{M}}_{p, \lambda, \alpha}(R)$ for all $1<p<\infty$ when $b \in B M O_{\alpha}(R)$ are proved.
\end{abstract}

AMS Subject Classification: 42B20, 42B25, 42B35

Key Words: commutator; generalized shift operator; Dunkl-type maximal function; Dunkl-type Morrey space; Dunkl-type modified Morrey space; $B M O_{\alpha}$ space

\section{Introduction}

In the theory of partial differential equations, together with weighted $L_{p, w}\left(R^{n}\right)$ spaces, the Morrey spaces $\mathcal{M}_{p, \lambda}\left(R^{n}\right)$ play an important role. The Morrey spaces were introduced by C.B. Morrey in 1938 in connection with certain problems in elliptic partial differential equations and calculus of variations (see [19]).

For $x \in R^{n}$ and $t>0$, let $B(x, t)$ denote the open ball centered at $x$ of radius $t$.

Received: February 22, 2020

(C) 2020 Academic Publications 
One of the most important variants of the Hardy-Littlewood maximal function defined by the formula

$$
M f(x)=\sup _{t>0}|B(x, t)|^{-1} \int_{B(x, t)}|f(y)| d y
$$

where $|B(x, t)|$ is the Lebesgue measure of the ball $B(x, t)$.

The operators $M_{\alpha}$ and $I_{\alpha}$ play important role in real and harmonic analysis (see, for example [23]).

Definition 1. Let $1 \leq p<\infty, 0 \leq \lambda \leq n$ and $[t]_{1}=\min \{1, t\}$. We denote by $\mathcal{M}_{p, \lambda}\left(R^{n}\right)$ Morrey space, and by $\widetilde{\mathcal{M}}_{p, \lambda}\left(R^{n}\right)$ the modified Morrey space, the set of locally integrable functions $f(x), x \in R^{n}$, with the finite norms

$$
\begin{aligned}
& \|f\|_{\mathcal{M}_{p, \lambda}}=\sup _{x \in R^{n}, t>0}\left(t^{-\lambda} \int_{B(x, t)}|f(y)|^{p} d y\right)^{1 / p}, \\
& \|f\|_{\widetilde{\mathcal{M}}_{p, \lambda}}=\sup _{x \in R^{n}, t>0}\left([t]_{1}^{-\lambda} \int_{B(x, t)}|f(y)|^{p} d y\right)^{1 / p},
\end{aligned}
$$

respectively.

Note that

$$
\begin{aligned}
& \widetilde{\mathcal{M}}_{p, 0}\left(R^{n}\right)=\mathcal{M}_{p, 0}\left(R^{n}\right)=L_{p}\left(R^{n}\right), \\
& \widetilde{\mathcal{M}}_{p, \lambda}\left(R^{n}\right)=\mathcal{M}_{p, \lambda}\left(R^{n}\right) \cap L_{p}\left(R^{n}\right)
\end{aligned}
$$

and if $\lambda<0$ or $\lambda>n$, then $\mathcal{M}_{p, \lambda}\left(R^{n}\right)=\widetilde{\mathcal{M}}_{p, \lambda}\left(R^{n}\right)=\Theta$, where $\Theta$ is the set of all functions equivalent to 0 on $R^{n}$.

These spaces appeared to be quite useful in the study of the local behaviour of the solutions to elliptic partial differential equations, apriori estimates and other topics in the theory of partial differential equations.

Definition 2. Let $1 \leq p<\infty, 0 \leq \lambda \leq n$. We denote by $W \mathcal{M}_{p, \lambda}\left(R^{n}\right)$ the weak Morrey space and by $W \widetilde{\mathcal{M}}_{p, \lambda}\left(R^{n}\right)$ the modified weak Morrey space, as the space of all functions $f \in W L_{p}^{l o c}\left(R^{n}\right)$ with finite norms

$$
\|f\|_{W \mathcal{M}_{p, \lambda}}=\sup _{r>0} r \sup _{x \in R^{n}, t>0}\left(t^{-\lambda}|\{y \in B(x, t):|f(y)|>r\}|\right)^{1 / p}
$$




$$
\|f\|_{W \widetilde{\mathcal{M}}_{p, \lambda}}=\sup _{r>0} r \sup _{x \in R^{n}, t>0}\left([t]_{1}^{-\lambda}|\{y \in B(x, t):|f(y)|>r\}|\right)^{1 / p},
$$

respectively.

Note that

$$
\begin{gathered}
W L_{p}\left(R^{n}\right)=W \mathcal{M}_{p, 0}\left(R^{n}\right)=W \widetilde{\mathcal{M}}_{p, 0}\left(R^{n}\right), \\
\mathcal{M}_{p, \lambda}\left(R^{n}\right) \subset W \mathcal{M}_{p, \lambda}\left(R^{n}\right) \text { and }\|f\|_{W \mathcal{M}_{p, \lambda}} \leq\|f\|_{\mathcal{M}_{p, \lambda}}, \\
\widetilde{\mathcal{M}}_{p, \lambda}\left(R^{n}\right) \subset W \widetilde{\mathcal{M}}_{p, \lambda}\left(R^{n}\right) \text { and }\|f\|_{W \widetilde{\mathcal{M}}_{p, \lambda}} \leq\|f\|_{\widetilde{\mathcal{M}}_{p, \lambda}}
\end{gathered}
$$

The commutator is defined for smooth functions $f$ by $[b, T] f=b T(f)-$ $T(b f)$, where $b$ is a locally integrable function on $R^{n}$ and $T$ is a CalderonZygmund operator. Coifman, Rochberg and Weiss [7] stated that $[b, T]$ is a bounded operator on $L_{p}\left(R^{n}\right), 1<p<\infty$, when $b$ is a $B M O$ function. Also, Chanillo [6] proved that commutators characterize Riesz potentials on the function space $B M O$.

\section{Definitions, notation and preliminaries}

Let $\alpha>-1 / 2$ be a fixed number and $\mu_{\alpha}$ be the weighted Lebesgue measure on $R$, given by

$$
d \mu_{\alpha}(x):=\left(2^{\alpha+1} \Gamma(\alpha+1)\right)^{-1}|x|^{2 \alpha+1} d x .
$$

For every $1 \leq p \leq \infty$, we denote by $L_{p, \alpha}(R)=L_{p}\left(R, d \mu_{\alpha}\right)$ the spaces of complex-valued functions $f$, measurable on $R$ such that

$$
\|f\|_{p, \alpha} \equiv\|f\|_{L_{p, \alpha}}=\left(\int_{R}|f(x)|^{p} d \mu_{\alpha}(x)\right)^{1 / p}<\infty \quad \text { if } \quad p \in[1, \infty),
$$

and

$$
\|f\|_{\infty, \alpha} \equiv\|f\|_{L_{\infty}}=\underset{x \in R}{e s s \sup }|f(x)| \quad \text { if } \quad p=\infty .
$$

For $1 \leq p<\infty$ we denote by $W L_{p, \alpha}(R)$, the weak $L_{p, \alpha}(R)$ spaces defined as the set of locally integrable functions $f$ with the finite norm

$$
\|f\|_{W L_{p, \alpha}}=\sup _{r>0} r\left(\mu_{\alpha}\{x \in R:|f(x)|>r\}\right)^{1 / p} .
$$

Note that

$$
L_{p, \alpha} \subset W L_{p, \alpha} \quad \text { and } \quad\|f\|_{W L_{p, \alpha}} \leq\|f\|_{p, \alpha} \text { for all } f \in L_{p, \alpha}(R) .
$$


Let $B(x, t)=\{y \in R:|y| \in] \max \{0,|x|-t\},|x|+t[\}$ and $B_{t} \equiv B(0, t)=$ ]$-t, t[, t>0$. Then

$$
\mu_{\alpha} B_{t}=b_{\alpha} t^{2 \alpha+2},
$$

where $b_{\alpha}=\left[2^{\alpha+1}(\alpha+1) \Gamma(\alpha+1)\right]^{-1}$.

Let $L_{p, \omega, \alpha}(R)$ be the space of measurable functions on $R$ with finite norm

$$
\|f\|_{L_{p, \omega, \alpha}}=\|f\|_{L_{p, \omega, \alpha}(R)}=\left(\int_{R}|f(x)|^{p} \omega(x) d \mu_{\alpha}(x)\right)^{1 / p}, \quad 1 \leq p<\infty
$$

and for $p=\infty$ the space $L_{\infty, \omega, \alpha}(R)=L_{\infty}(R)$.

Definition 3. The weight function $\omega$ belongs to the class $A_{p, \alpha}(R)$ for $1 \leq p<\infty$, if the following statement

$$
\int_{B(x, r)} \omega(y) d \mu_{\alpha}(y)\left(\int_{B(x, r)} \omega^{-\frac{1}{p-1}}(y) d \mu_{\alpha}(y)\right)^{p-1} \leq\left(\mu_{\alpha} B(x, r)\right)^{p}
$$

is finite and $\omega$ belongs to $A_{1, \alpha}(R)$, if there exists a positive constant $C$ such that for any $x \in R$ and $r>0$

$$
\frac{1}{\mu_{\alpha} B(x, r)} \int_{B(x, r)} \omega(y) d \mu_{\alpha}(y) \leq C \operatorname{ess}_{y \in B(x, r)} \frac{1}{\omega(y)} .
$$

Definition 4. Let $1 \leq p<\infty, 0 \leq \lambda \leq 2 \alpha+2$. We denote by $\mathcal{M}_{p, \lambda, \alpha}(R)$ Dunkl-type Morrey space ( $\equiv D$-Morrey space) as the set of locally integrable functions $f(x), x \in R$, with the finite norm

$$
\|f\|_{\mathcal{M}_{p, \lambda, \alpha}}=\sup _{t>0, x \in R}\left(t^{-\lambda} \int_{B_{t}}\left[\tau_{x}|f(y)|\right]^{p} d \mu_{\alpha}(y)\right)^{1 / p} .
$$

Definition 5. Let $1 \leq p<\infty, 0 \leq \lambda \leq 2 \alpha+2$ and $[t]_{1}=\min \{1, t\}$. We denote by $\widetilde{\mathcal{M}}_{p, \lambda, \alpha}(R)$ the Dunkl-type modified Morrey space as the set of locally integrable functions $f(x), x \in R$, with finite norm

$$
\|f\|_{\widetilde{\mathcal{M}}_{p, \lambda, \alpha}}=\sup _{t>0, x \in R}\left([t]_{1}^{-\lambda} \int_{B_{t}}\left[\tau_{x}|f(y)|\right]^{p} d \mu_{\alpha}(y)\right)^{1 / p} .
$$

Definition 6. Let $1 \leq p<\infty$ and $0 \leq \lambda \leq 2 \alpha+2$. A measurable function $f$ on $R$ is said to belong to the Dunkl-type modified weak Morrey space $W \widetilde{\mathcal{M}}_{p, \lambda, \alpha}(R)$ if the quasi-norm 


$$
\|f\|_{W \widetilde{\mathcal{M}}_{p, \lambda, \alpha}}=\sup _{r>0} r \sup _{t>0, x \in R}\left([t]_{1}^{-\lambda} \int_{\left\{y \in B_{t}: \tau_{x}|f(y)|\right\}} d \mu_{\alpha}(y)\right)^{1 / p}
$$

is finite.

Let $M_{\alpha}^{\sharp}$ be the Dunkl-type sharp maximal function defined by

$$
M_{\alpha}^{\sharp} f(x)=\sup _{r>0} \frac{1}{\mu_{\alpha} B_{r}} \int_{B_{r}}\left|\tau_{x} f(y)-f_{B_{r}}(x)\right| d \mu_{\alpha}(y),
$$

where $f_{B_{r}}(x)=\frac{1}{\mu_{\alpha} B_{r}} \int_{B_{r}} \tau_{x} f(y) d \mu_{\alpha}(y)$.

We denote by $B M O_{\alpha}(R)$ (Dunkl-type $B M O$ space) the set of locally integrable functions $f$ with finite norm

$$
\|f\|_{B M O_{\alpha}}=\sup _{r>0, x \in R} \frac{1}{\mu_{\alpha} B_{r}} \int_{B_{r}}\left|\tau_{x} f(y)-f_{B_{r}}(x)\right| d \mu_{\alpha}(y)<\infty,
$$

or

$$
\|f\|_{B M O_{\alpha}}=\inf _{C} \sup _{r>0, x \in R} \frac{1}{\mu_{\alpha} B_{r}} \int_{B_{r}}\left|\tau_{x} f(y)-C\right| d \mu_{\alpha}(y) .
$$

Theorem 7. ([17]) 1) Let $f \in L_{1, \alpha}^{\text {loc }}(R)$. If

$$
\sup _{t>0, x \in R}\left(\mu_{\alpha}\left(B_{t}\right)^{-1} \int_{B_{t}}\left|\tau_{y} f(x)-f_{B_{t}}\right|^{p} d \mu_{\alpha}(y)\right)^{1 / p}=\|f\|_{B M O_{p, \alpha}}<\infty
$$

then for any $1<p<\infty$,

$$
\|f\|_{B M O_{\alpha}} \leq\|f\|_{B M O_{p, \alpha}} \leq A_{p}\|f\|_{B M O_{\alpha}},
$$

where the constant $A_{p}$ depends only on $p$.

2) Let $f \in B M O_{\alpha}(R)$. Then, there is a constant $C>0$ such that

$$
\left|f_{B_{r}}-f_{B_{t}}\right| \leq C\|f\|_{B M O_{\alpha}} \ln \frac{t}{r}, \quad 0<2 r<t,
$$

where $C$ is independent of $f, x, r$ and $t$.

For all $x, y, z \in R$, we put

$$
W_{\alpha}(x, y, z)=\left(1-\sigma_{x, y, z}+\sigma_{z, x, y}+\sigma_{z, y, x}\right) \Delta_{\alpha}(x, y, z)
$$


where

$$
\sigma_{x, y, z}=\left\{\begin{array}{cc}
\frac{x^{2}+y^{2}-z^{2}}{2 x y} & \text { if } x, y \in R \backslash\{0\}, \\
0 & \text { otherwise, }
\end{array}\right.
$$

and $\Delta_{\alpha}$ is the Bessel kernel given by

$$
\Delta_{\alpha}(x, y, z)=\left\{\begin{array}{cl}
d_{\alpha} \frac{\left(\left[(|x|+|y|)^{2}-z^{2}\right]\left[z^{2}-(|x|-|y|)^{2}\right]\right)^{\alpha-1 / 2}}{|x y z|^{2 \alpha}}, & \text { if }|z| \in A_{x, y} \\
0, & \text { otherwise }
\end{array}\right.
$$

where $d_{\alpha}=(\Gamma(\alpha+1))^{2} /\left(2^{\alpha-1} \sqrt{\pi} \Gamma\left(\alpha+\frac{1}{2}\right)\right)$ and $A_{x, y}=[|| x|-| y||,|x|+|y|]$.

Properties 8. (see Rösler [24]) The signed kernel $W_{\alpha}$ is even with respect to all variables and satisfies the following properties

$$
\begin{gathered}
W_{\alpha}(x, y, z)=W_{\alpha}(y, x, z)=W_{\alpha}(-x, z, y), \\
W_{\alpha}(x, y, z)=W_{\alpha}(-z, y,-x)=W_{\alpha}(-x,-y,-z)
\end{gathered}
$$

and

$$
\int_{R}\left|W_{\alpha}(x, y, z)\right| d \mu_{\alpha}(z) \leq 4
$$

In the sequel we consider the signed measure $\nu_{x, y}$, on $R$, given by

$$
\nu_{x, y}=\left\{\begin{array}{cl}
W_{\alpha}(x, y, z) d \mu_{\alpha}(z) & \text { if } x, y \in R \backslash\{0\}, \\
d \delta_{x}(z) & \text { if } y=0 \\
d \delta_{y}(z) & \text { if } x=0 .
\end{array}\right.
$$

Definition 9. For $x, y \in R$ and $f$ a continuous function on $R$, we put

$$
\tau_{x} f(y)=\int_{R} f(z) d \nu_{x, y}(z) .
$$

The operators $\tau_{x}, x \in R$, are called Dunkl translation operators on $R$ and it can be expressed in the following form (see [24])

$$
\begin{gathered}
\tau_{x} f(y)=c_{\alpha} \int_{0}^{\pi} f_{e}\left((x, y)_{\theta}\right) h_{1}(x, y, \theta)(\sin \theta)^{2 \alpha} d \theta \\
+c_{\alpha} \int_{0}^{\pi} f_{o}\left((x, y)_{\theta}\right) h_{2}(x, y, \theta)(\sin \theta)^{2 \alpha} d \theta
\end{gathered}
$$


where $(x, y)_{\theta}=\sqrt{x^{2}+y^{2}-2|x y| \cos \theta}, f=f_{e}+f_{o}, f_{o}$ and $f_{e}$ being respectively the odd and the even parts of $f$, with

$$
\begin{gathered}
c_{\alpha} \equiv\left(\int_{0}^{\pi}(\sin \theta)^{2 \alpha} d \theta\right)^{-1}=\frac{\Gamma(\alpha+1)}{\sqrt{\pi} \Gamma(\alpha+1 / 2)}, \\
h_{1}(x, y, \theta)=1-\operatorname{sgn}(x y) \cos \theta
\end{gathered}
$$

and

$$
h_{2}(x, y, \theta)= \begin{cases}\frac{(x+y)[1-\operatorname{sgn}(x y) \cos \theta]}{(x, y)_{\theta}}, & \text { if } x y \neq 0 \\ 0, & \text { if } x y=0\end{cases}
$$

Using the change of variable $z=(x, y)_{\theta}$, we have also (see [3])

$$
\begin{aligned}
\tau_{x} f(y)=c_{\alpha} \int_{0}^{\pi} & \left\{f\left((x, y)_{\theta}\right)+f\left(-(x, y)_{\theta}\right)\right. \\
& \left.+\frac{x+y}{(x, y)_{\theta}}\left[f\left((x, y)_{\theta}\right)-f\left(-(x, y)_{\theta}\right)\right]\right\}(1-\cos \theta)(\sin \theta)^{2 \alpha} d \theta
\end{aligned}
$$

Now we define the Dunkl-type maximal function by

$$
M_{\alpha} f(x)=\sup _{r>0}\left(\mu_{\alpha} B_{r}\right)^{-1} \int_{B_{r}} \tau_{x}|f|(y) d \mu_{\alpha}(y) .
$$

Theorem 10. ([11])

1. If $f \in L_{1, \omega, \alpha}(R)$ and $\omega \in A_{1, \alpha}(R)$, then $M_{\alpha} f \in W L_{1, \omega, \alpha}(R)$ and

$$
\left\|M_{\alpha} f\right\|_{W L_{1, \omega, \alpha}} \leq C_{1, \alpha}\|f\|_{L_{1, \omega, \alpha}},
$$

where $C_{1, \alpha}$ depends only on $\alpha$.

2. If $f \in L_{p, \omega, \alpha}(R), 1<p<\infty$ and $\omega \in A_{p, \alpha}(R)$, then $M_{\alpha} f \in L_{p, \omega, \alpha}(R)$ and

$$
\left\|M_{\alpha} f\right\|_{L_{p, \omega, \alpha}} \leq C_{p, \alpha}\|f\|_{L_{p, \omega, \alpha}}
$$

where $C_{p, \alpha}$ depends only on $p, \alpha$.

Theorem 11. ([13])

1. If $f \in \mathcal{M}_{1, \lambda, \alpha}(R), 0 \leq \lambda<2 \alpha+2$, then $M_{\alpha} f \in W \mathcal{M}_{1, \lambda, \alpha}(R)$ and

$$
\left\|M_{\alpha} f\right\|_{W \mathcal{M}_{1, \lambda, \alpha}} \leq C_{1, \lambda, \alpha}\|f\|_{\mathcal{M}_{1, \lambda, \alpha}}
$$

where $C_{1, \lambda, \alpha}$ depends only on $\lambda, \alpha$ and $n$.

2. If $f \in \mathcal{M}_{p, \lambda, \alpha}(R), 1<p<\infty, 0 \leq \lambda<2 \alpha+2$, then $M_{\alpha} f \in \mathcal{M}_{p, \lambda, \alpha}(R)$ and

$$
\left\|M_{\alpha} f\right\|_{\mathcal{M}_{p, \lambda, \alpha}} \leq C_{p, \lambda, \alpha}\|f\|_{\mathcal{M}_{p, \lambda, \alpha}}
$$

where $C_{p, \lambda, \alpha}$ depends only on $p, \lambda, \alpha$ and $n$. 
For a real parameter $\alpha \geq-1 / 2$, we consider the Dunkl operator, associated with the reflection group $Z_{2}$ on $R$ :

$$
\Lambda_{\alpha}(f)(x)=\frac{d}{d x} f(x)+\frac{2 \alpha+1}{x}\left(\frac{f(x)-f(-x)}{2}\right) .
$$

Note that $\Lambda_{-1 / 2}=d / d x$.

For $\alpha \geq-1 / 2$ and $\lambda \in C$, the initial value problem:

$$
\Lambda_{\alpha}(f)(x)=\lambda f(x), \quad f(0)=1, \quad x \in R
$$

has a unique solution $E_{\alpha}(\lambda x)$ called Dunkl kernel [8, 20, 26] and given by

$$
E_{\alpha}(\lambda x)=j_{\alpha}(i \lambda x)+\frac{\lambda x}{2(\alpha+1)} j_{\alpha+1}(i \lambda x), \quad x \in R
$$

where $j_{\alpha}$ is the normalized Bessel function of the first kind and order $\alpha$ [27], defined by

$$
j_{\alpha}(z)=2^{\alpha} \Gamma(\alpha+1) \frac{J_{\alpha}(z)}{z^{\alpha}}=\Gamma(\alpha+1) \sum_{n=0}^{\infty} \frac{(-1)^{n}(z / 2)^{2 n}}{n ! \alpha(n+\alpha+1)}, \quad z \in C .
$$

We can write for $x \in R$ and $\lambda \in C$ (see Rösler [24], p. 295)

$$
E_{\alpha}(-i \lambda x)=\frac{\Gamma(\alpha+1)}{\sqrt{\pi} \Gamma(\alpha+1 / 2)} \int_{-1}^{1}\left(1-t^{2}\right)^{\alpha-1 / 2}(1-t) e^{i \lambda x t} d t .
$$

Note that $E_{-1 / 2}(\lambda x)=e^{\lambda x}$.

The Dunkl transform $\mathcal{F}_{\alpha}$ of a function $f \in L_{1, \alpha}(R)$, is given by

$$
\mathcal{F}_{\alpha} f(\lambda):=\int_{R} E_{\alpha}(-i \lambda x) f(x) d \mu_{\alpha}(x), \quad \lambda \in R
$$

Here the integral makes sense since $\mid E_{\alpha}(i x \mid \leq 1$ for every $x \in R$ [24], p. 295.

Note that $\mathcal{F}_{-1 / 2}$ agrees with the classical Fourier transform $\mathcal{F}$, given by:

$$
\mathcal{F} f(\lambda):=(2 \pi)^{-1 / 2} \int_{R} e^{-i \lambda x} f(x) d x, \quad \lambda \in R .
$$

Proposition 12. (see Soltani [21])

(i) If $f$ is an even positive continuous function, then $\tau_{x} f$ is positive.

(ii) For all $x \in R$ the operator $\tau_{x}$ extends to $L_{p, \alpha}(R), p \geq 1$ and we have for $f \in L_{p, \alpha}(R)$,

$$
\left\|\tau_{x} f\right\|_{p, \alpha} \leq 4\|f\|_{p, \alpha} .
$$

(iii) For all $x, \lambda \in R$ and $f \in L_{1, \alpha}(R)$, we have

$$
\mathcal{F}_{\alpha}\left(\tau_{x} f\right)(\lambda)=E_{\alpha}(i \lambda x) \mathcal{F}_{\alpha} f(\lambda) .
$$


Let $f$ and $g$ be two continuous functions on $R$ with compact support. We define the generalized convolution $*_{\alpha}$ of $f$ and $g$ by

$$
f *_{\alpha} g(x):=\int_{R} \tau_{x} f(-y) g(y) d \mu_{\alpha}(y), \quad x \in R .
$$

The generalized convolution $*_{\alpha}$ is associative and commutative [24]. Note that $*_{-1 / 2}$ agrees with the standard convolution $*$.

Proposition 13. (see Soltani [21])

(i) If $f$ is an even positive function and $g$ a positive function with compact support, then $f *_{\alpha} g$ is positive.

(ii) Assume that $p, q, r \in[1,+\infty$ [ satisfying $1 / p+1 / q=1+1 / r$ (the Young condition). Then the map $(f, g) \mapsto f *_{\alpha} g$, defined on $\mathcal{E}_{c} \times \mathcal{E}_{c}$, extends to a continuous map from $L_{p, \alpha}(R) \times L_{q, \alpha}(R)$ to $L_{r, \alpha}(R)$, and we have

$$
\left\|f *_{\alpha} g\right\|_{r, \alpha} \leq 4\|f\|_{p, \alpha}\|g\|_{q, \alpha} .
$$

(ii) For all $f \in L_{1, \alpha}(R)$ and $g \in L_{2, \alpha}(R)$, we have

$$
\mathcal{F}_{\alpha}\left(f *_{\alpha} g\right)=\left(\mathcal{F}_{\alpha} f\right)\left(\mathcal{F}_{\alpha} g\right)
$$

\section{Main results and proofs}

\subsection{Maximal commutators in Dunkl-type modified Morrey spaces}

The commutator generated by the Dunkl-type maximal operator $M_{\alpha}$ for a given measurable function $b$ is formally defined by

$$
\left[M_{\alpha}, b\right] f=M_{\alpha}(b f)-b M_{\alpha}(f)
$$

and for a given measurable function $b$, the Dunkl-type maximal commutator is defined by

$$
M_{b, \alpha}(f)(x):=\sup _{r>0} \frac{1}{\mu_{\alpha} B_{r}} \int_{B_{r}} \tau_{y}|(b(x)-b(y)) f(x)| d \mu_{\alpha}(y)
$$

for all $x \in R$. 
Lemma 14. Let $1<s<\infty$ and $b \in B M O(R)$. Then there exists $C>0$ such that for all $x \in R$

$$
M_{\alpha}^{\sharp}\left(M_{b, \alpha} f\right)(x) \leq C\|b\|_{B M O_{\alpha}}\left(\left(M_{\alpha}\left(M_{\alpha} f\right)^{s}\right)^{\frac{1}{s}}(x)+M_{\alpha}\left(M_{\alpha}|f|^{s}\right)^{\frac{1}{s}}(x)\right)
$$

holds.

Proof. From the boundedness of the Dunkl-type maximal operator $M_{\alpha}$ and the pointwise inequality we have

$$
M_{\alpha}^{\sharp}\left(M_{b, \alpha} f\right)(x) \leq 2 M_{\alpha}\left(M_{b, \alpha} f\right)(x), x \in R .
$$

Since $M_{b, \alpha}(f)(y)=\sup _{t>0} M_{b, t, \gamma}(f)(y)$ we get

$$
\begin{gathered}
M_{b, t, \gamma}(f)(y)=\frac{1}{\mu_{\alpha} B_{t}} \int_{B_{t}} \tau_{y}(|b(y)-b(z)||f(z)|) d \mu_{\alpha}(z) \\
\leq \frac{1}{\mu_{\alpha} B_{t}} \int_{B_{t}} \tau_{y}\left(\left|b(z)-b_{B_{t}}\right||f(z)|\right) d \mu_{\alpha}(z) \\
+\left|b(y)-b_{B_{t}}\right| \frac{1}{\mu_{\alpha} B_{t}} \int_{B_{t}} \tau_{y}[|f|](z) d \mu_{\alpha}(z) \\
\leq \frac{1}{\mu_{\alpha} B_{t}}\left(\int_{B_{t}} \tau_{y}\left|b(z)-b_{B_{t}}\right|^{s^{\prime}} d \mu_{\alpha}(z)\right)^{\frac{1}{s^{\prime}}}\left(\int_{B_{t}} \tau_{y}[|f|]^{s}(z) d \mu_{\alpha}(z)\right)^{\frac{1}{s}} \\
+\left|b(y)-b_{B_{t}}\right| \frac{1}{\mu_{\alpha} B_{t}} \int_{B_{t}} \tau_{y}[|f|](z) d \mu_{\alpha}(z) \\
\leq C\|b\|_{B M O_{\alpha}}\left(M_{\alpha}|f|^{s}\right)^{\frac{1}{s}}(y)+\left|b(y)-b_{B_{t}}\right| \frac{1}{\mu_{\alpha} B_{t}} \int_{B_{t}} \tau_{y}[|f|](z) d \mu_{\alpha}(z) .
\end{gathered}
$$

From the Hölder inequality we have

$$
\begin{aligned}
& \frac{1}{\mu_{\alpha} B_{r}} \int_{B_{r}} \tau_{x}\left[\left|b(y)-b_{B_{t}}\right| \frac{1}{\mu_{\alpha} B_{t}}\left(\int_{B_{t}} \tau_{y}[|f|](z) d \mu_{\alpha}(z)\right)\right] d \mu_{\alpha}(y) \\
\leq & \frac{1}{\mu_{\alpha} B_{r}}\left(\int_{B_{r}} \tau_{x}\left|b(y)-b_{B_{r}}\right|^{s^{\prime}} d \mu_{\alpha}(y)\right)^{\frac{1}{s^{\prime}}}\left(\int_{B_{r}} \tau_{x}\left(M_{\alpha} f\right)^{s}(y) d \mu_{\alpha}(y)\right)^{\frac{1}{s}}
\end{aligned}
$$




$$
\begin{gathered}
+\frac{1}{\mu_{\alpha} B_{r}} \int_{B_{r}} \tau_{x}\left[\left|b_{B_{t}}-b_{B_{r}}\right| M_{\alpha} f(y)\right] d \mu_{\alpha}(y) \\
\leq C\|b\|_{B M O_{\alpha}}\left(M_{\alpha}\left(M_{\alpha} f\right)^{s}\right)^{\frac{1}{s}}(x) .
\end{gathered}
$$

Therefore we get

$$
\begin{gathered}
M_{\alpha}\left(M_{b, \alpha} f\right)(x)=\sup _{r>0} \frac{1}{\mu_{\alpha} B_{r}} \int_{B_{r}} \tau_{x}\left[M_{b, \alpha} f\right](y) d \mu_{\alpha}(y) \\
\leq C\|b\|_{B M O_{\alpha}}\left(\left(M_{\alpha}\left(M_{\alpha} f\right)^{s}\right)^{\frac{1}{s}}(x)\right. \\
\left.+\sup _{r>0} \frac{1}{\mu_{\alpha} B_{r}} \int_{B_{r}} \tau_{x}\left(M_{\alpha}|f|^{s}\right)^{\frac{1}{s}}(y) d \mu_{\alpha}(y)\right) \\
\leq C\|b\|_{B M O_{\alpha}}\left(\left(M_{\alpha}\left(M_{\alpha} f\right)^{s}\right)^{\frac{1}{s}}(x)+M_{\alpha}\left(M_{\alpha}|f|^{s}\right)^{\frac{1}{s}}(x)\right) .
\end{gathered}
$$

Thus Lemma 14 is proved.

Proposition 3.1. ([15]) For all weights $\omega$ and all nonnegative function $f$ satisfying $\nu(\{x \in X: f(x)>\beta\})<\infty$ for all $\beta>0$, there exists a positive constant $C$ such that

1. If $\nu(X)=\infty$, then

$$
\int_{X} f(y) g(y) d \nu(y) \leq C \int_{X} M^{\sharp} f(y) M g(y) d \nu(y) .
$$

2. If $\nu(X)<\infty$, then

$$
\int_{X} f(y) g(y) d \nu(y) \leq C \int_{X} M^{\sharp} f(y) M g(y) d \nu(y)+C g(X) \nu_{X}(f),
$$

where $g$ is nonnegative function, $g(X)=\int_{X} g(x) d \nu(x), \nu_{X}(f)=\frac{1}{\nu(X)} \int_{X} f(y) d \nu(y)$.

Lemma 15. Let $1<p<\infty$ and $\omega \in A_{p, \alpha}(R)$. Then

$$
\left\|f \omega^{\frac{1}{p}}\right\|_{L_{p, \alpha}} \leq C\left\|\omega^{\frac{1}{p}} M_{\alpha}^{\sharp} f\right\|_{L_{p, \alpha}},
$$

where the constant $C>0$ is independent of $f$. 
Proof. Let $(X, \nu)$ be a homogeneous type space. According to Proposition 3.1, we have

$$
\begin{array}{r}
\left\|f \omega^{\frac{1}{p}}\right\|_{L_{p, \alpha}} \leq C \sup _{\|g\|_{L_{p^{\prime}, \alpha}} \leq 1}\left|\int_{R} f(y) g(y) \omega^{\frac{1}{p}}(y) d \mu_{\alpha}(y)\right| \\
=C \sup _{\|g\|_{L_{p^{\prime}} \leq 1} \mid}\left|\int_{X} f(y) g(y) \omega^{\frac{1}{p}}(y) d \nu(y)\right| \\
\leq C \sup _{\|g\|_{L_{p^{\prime}} \leq 1}}\left|\int_{X} M^{\sharp} f(y) M\left(g \omega^{\frac{1}{p}}\right)(y) d \nu(y)\right| .
\end{array}
$$

Hence,

$$
\left\|f \omega^{\frac{1}{p}}\right\|_{L_{p, \alpha}} \leq C \sup _{\|g\|_{L_{p^{\prime}, \alpha}} \leq 1}\left|\int_{R} M_{\alpha}^{\sharp} f(y) M_{\alpha}\left(g \omega^{\frac{1}{p}}\right)(y) d \mu_{\alpha}(y)\right| .
$$

Finally by using the Hölder inequality and Theorem 10, we get

$$
\begin{gathered}
\left\|f \omega^{\frac{1}{p}}\right\|_{L_{p, \alpha}} \leq C \sup _{\|g\|_{L_{p^{\prime}, \alpha}} \leq 1}\left\|\omega^{\frac{1}{p}} M_{\alpha}^{\sharp} f\right\|_{L_{p, \alpha}}\left\|\omega^{-\frac{1}{p}} M_{\alpha}\left(g \omega^{\frac{1}{p}}\right)\right\|_{L_{p^{\prime}, \alpha}} \\
\quad \leq C \sup _{\|g\|_{L_{p^{\prime}, \alpha} \leq 1}}\left\|\omega^{\frac{1}{p}} M_{\alpha}^{\sharp} f\right\|_{L_{p, \alpha}}\|g\|_{L_{p^{\prime}, \alpha}} \leq C\left\|\omega^{\frac{1}{p}} M_{\alpha}^{\sharp} f\right\|_{L_{p, \alpha}} .
\end{gathered}
$$

Thus Lemma 15 is proved.

Theorem 16. Let $b \in B M O_{\alpha}(R), 1<p<\infty$ and $\omega \in A_{p, \alpha}(R)$. Then $M_{b, \alpha}$ is bounded on the space $L_{p, \omega, \alpha}(R)$.

Proof. By using Lemma 14, Lemma 15 and Theorem 10, we have $M_{b, \alpha}$ is bounded on the space $L_{p, \omega, \alpha}(R)$.

The operators $M_{b, \alpha}$ and $\left[M_{\alpha}, b\right]$ are essentially different from each other. For example, $M_{b, \alpha}$ is a positive and sublinear operator, but $\left[M_{\alpha}, b\right]$ is neither positive nor sublinear. However, if $b$ satisfies some additional conditions, then the operator $M_{b, \alpha}$ is controlled by $\left[M_{\alpha}, b\right]$.

Theorem 17. Let $1<p<\infty$ and $0 \leq \lambda<2 \alpha+2$. Then the commutator $M_{b, \alpha}$ is bounded on $\widetilde{\mathcal{M}}_{p, \lambda, \alpha}(R)$ if and only if $b \in B M O_{\alpha}(R)$. 
Proof. Sufficiency: Let $1<p<\infty, 0 \leq \lambda<2 \alpha+2, f \in \widetilde{\mathcal{M}}_{p, \lambda, \alpha}(R), x \in R$. We have

$$
\int_{B_{t}} \tau_{y}\left[M_{b, \alpha} f\right]^{p}(x) d \mu_{\alpha}(y) \leq \int_{R} \tau_{y}\left[M_{b, \alpha} f\right]^{p}(x)\left(M_{\alpha} \chi_{B_{t}}(y)\right)^{\delta} d \mu_{\alpha}(y) .
$$

Taking into account the properties of $A_{p, \alpha}(R)$ we can easily see that $\left(M_{\alpha} \chi_{B_{t}}\right)^{\delta} \in$ $A_{p, \alpha}(R)$ for any $0<\delta<1$. Then by using Lemma 15 and Theorem 16 we obtain

$$
\begin{aligned}
& \int_{B_{t}} \tau_{y}\left[M_{b, \alpha} f\right]^{p}(x) d \mu_{\alpha}(y) \\
& \leq C\|b\|_{B M O_{\alpha}}^{p} \int_{R} \tau_{y}[|f|]^{p}(x)\left(M_{\alpha} \chi_{B_{r}}(y)\right)^{\theta} d \mu_{\alpha}(y) \\
& \leq C\|b\|_{B M O_{\alpha}}^{p} \int_{B_{r}} \tau_{y}[|f|]^{p}(x) d \mu_{\alpha}(y) \\
& +C\|b\|_{B M O_{\alpha}}^{p} \sum_{j=1_{B_{2} j+1_{r} \backslash B_{2^{j}}}^{\infty}}^{\infty} \tau_{y}[|f|]^{p}(x)\left(M_{\alpha} \chi_{B_{r}}(y)\right)^{\theta} d \mu_{\alpha}(y) \\
& \leq C\|b\|_{B M O_{\alpha}}^{p} \int_{B_{r}} \tau_{y}[|f|]^{p}(x) d \mu_{\alpha}(y) \\
& +C\|b\|_{B M O_{\alpha}}^{p} \sum_{j=1_{B_{2^{j+1}} \backslash B_{2^{j}}}}^{\infty} \int_{y}[|f|]^{p}(x) \frac{r^{(2 \alpha+2) \theta}}{(|y|+r)^{(2 \alpha+2) \theta}} d \mu_{\alpha}(y) \\
& \leq C\|b\|_{B M O_{\alpha}}^{p}\|f\|_{\widetilde{\mathcal{M}}_{p, \lambda, \alpha}}^{p}\left([r]_{1}^{\lambda}+\sum_{j=1}^{\infty} \frac{1}{\left(2^{j}+1\right)^{(2 \alpha+2) \theta}}\left[2^{j+1} r\right]_{1}^{\lambda}\right) \\
& \leq C[r]_{1}^{\lambda}\|b\|_{B M O_{\alpha}}^{p}\|f\|_{\widetilde{\mathcal{M}}_{p, \lambda, \alpha}}^{p} .
\end{aligned}
$$

Necessity: Let $M_{b, \alpha}$ be bounded from $\widetilde{\mathcal{M}}_{p, \lambda, \alpha}(R)$ to $\widetilde{\mathcal{M}}_{p, \lambda, \alpha}(R)$, $1<p<\infty$.

Obviously,

$$
\|f\|_{\widetilde{\mathcal{M}}_{p, \lambda, \alpha}}=\sup _{t>0, x \in R}\left([t]_{1}^{-\lambda} \int_{B_{t}} \tau_{y}[|f|]^{p}(x) d \mu_{\alpha}(y)\right)^{1 / p}
$$


Now we consider $f=\chi_{B_{r}}$. It is easy to compute that

$$
\begin{aligned}
\left\|\chi_{B_{r}}\right\|_{\widetilde{\mathcal{M}}_{p, \lambda, \alpha}} & \approx \sup _{t>0, x \in R}\left([t]_{1}^{-\lambda} \int_{B_{t}} \tau_{y} \chi_{B_{r}}(x) d \mu_{\alpha}(y)\right)^{1 / p} \\
& \approx \sup _{t>0, x \in R}\left([t]_{1}^{-\lambda} \int_{E(x, t)} \chi_{B_{r}}(y) d \mu_{\alpha}(y)\right)^{1 / p} \\
& \approx \sup _{B(x, t) \subset B_{r}}\left([t]_{1}^{-\lambda}\left|B(x, t) \cap B_{r}\right|_{\gamma}\right)^{1 / p} \leq[r]_{1}^{\frac{-\lambda}{p}} r^{\frac{2 \alpha+2}{p}} .
\end{aligned}
$$

Then

$$
\begin{aligned}
& \frac{1}{\mu_{\alpha} B_{t}} \int_{B_{t}}\left|\tau_{z} b(x)-f_{B_{t}}\right| d \mu_{\alpha}(z) \\
= & \frac{1}{\mu_{\alpha} B_{t}} \int_{B_{t}}\left|\tau_{z} b(x)-\frac{1}{\mu_{\alpha} B_{t}} \int_{B_{t}} \tau_{z} b(y) d \mu_{\alpha}(y)\right| d \mu_{\alpha}(z) \\
\leq & \frac{1}{\mu_{\alpha} B_{t}} \int_{B_{t}} \frac{1}{\mu_{\alpha} B_{t}} \int_{B_{t}}\left|\tau_{z} b(x)-\tau_{z} b(y)\right| d \mu_{\alpha}(y) d \mu_{\alpha}(z) \\
\leq & \frac{1}{\mu_{\alpha} B_{t}} \int_{B_{t}} \frac{1}{\mu_{\alpha} B_{t}} \int_{B_{t}}\left|\tau_{z}(b(x)-b(y))\right| d \mu_{\alpha}(y) d \mu_{\alpha}(z) \\
\leq & \frac{1}{\mu_{\alpha} B_{t}} \int_{B_{t}} M_{b, \alpha} \chi_{B_{t}}(z) \mu_{\alpha}(z) \\
\leq & C t^{-2 \alpha-2}[t]_{1}^{\lambda}\left\|M_{b, \alpha} \chi_{B_{t}}\right\|_{\widetilde{\mathcal{M}}_{p, \lambda, \alpha}}\left\|\chi_{B_{t}}\right\| \|_{\tilde{L}_{p^{\prime}, \lambda, \gamma}} \\
\leq & C[t]_{1}^{\frac{-\lambda}{p}+\frac{-\lambda}{p^{\prime}}+\lambda} t^{\frac{2 \alpha+2}{p^{\prime}}+\frac{2 \alpha+2}{p}-2 \alpha-2} \leq C .
\end{aligned}
$$

Thus Theorem 17 is proved.

Theorem 18. Let $0 \leq \lambda<2 \alpha+2$ and $b \in B M O_{\alpha}(R)$. Then the commutator $M_{b, \alpha}$ is bounded from $\widetilde{\mathcal{M}}_{1, \lambda, \alpha}(R)$ to $W \widetilde{\mathcal{M}}_{1, \lambda, \alpha}(R)$.

Proof. Let $0 \leq \lambda<2 \alpha+2$ and $f \in \widetilde{\mathcal{M}}_{1, \lambda, \alpha}(R)$. This assertion is easily obtained from the inequality $f(x) \leq M_{\alpha} f(x)$. Finally, by using (3.1) and Theorem 10, we get

$$
\left\|M_{b, \alpha} f\right\|_{W \widetilde{\mathcal{M}}_{1, \lambda, \alpha}} \leq\left\|M_{\alpha}\left(M_{b, \alpha} f\right)\right\|_{W \widetilde{\mathcal{M}}_{1, \lambda, \alpha}}
$$




$$
\begin{gathered}
\leq\|b\|_{B M O_{\alpha}}\left\|\left(M_{\alpha}\left(M_{\alpha} f\right)^{s}\right)^{\frac{1}{s}}+M_{\alpha}\left(M_{\alpha}|f|^{s}\right)^{\frac{1}{s}}\right\|_{W \widetilde{\mathcal{M}}_{1, \lambda, \alpha}} \\
\leq\|b\|_{B M O_{\alpha}}\|f\|_{\widetilde{\mathcal{M}}_{1, \lambda, \alpha}} .
\end{gathered}
$$

\section{References}

[1] C. Abdelkefi and M. Sifi, Dunkl translation and uncentered maximal operator on the real line, Intern. J. Math. and Math. Sciences, 2007 (2007), Art. ID 87808, 9 pp.; doi:10.1155/2007/87808.

[2] C. Abdelkefi and M. Sifi, On the uniform convergence of partial Dunkl integrals in Besov-Dunkl spaces, Fract. Calc. Appl. Anal., 9, No 1 (2006), 43-56.

[3] C. Abdelkefi and M. Sifi, Characterization of Besov spaces for the Dunkl operator on the real line, J. of Inequalities in Pure and Appl. Math., 8, No 3 (2007), Art. 73, 1-21.

[4] D.R. Adams, A note on Riesz potentials, Duke Math., 42 (1975), 765-778.

[5] C. Aykol, H. Armutcu and M.N. Omarova, Maximal commutator and commutator of maximal function on modified Morrey spaces, Trans. of NAS of Azerbaijan, Issue Mathematics, 36, No 1 (2016), 29-35.

[6] S. Chanillo, A note on commutators, Indiana Math. J., 31 (1982), 7-16.

[7] R. Coifman, R. Rochberg and G. Weiss, Factorization theorems for Hardy spaces in several variables, Ann. of Math., 103, No 3 (1976), 611-635.

[8] C.F. Dunkl, Differential-difference operators associated with reflections groups, Trans. Amer. Math. Soc., 311 (1989), 167-183.

[9] A. Gogatishvili, R.Ch. Mustafayev and M. Agcayazi, Weak-type estimates in Morrey spaces for maximal commuatator and commutator of maximal function, Tokyo J. of Mathematics, 41, No 1 (2018), 193-218.

[10] V.S. Guliyev, J. Hasanov and Y. Zeren, Necessary and sufficient conditions for the boundedness of the Riesz potential in modified Morrey spaces $J$. Math. Inequal., 5, No 4 (2011), 491-506. 
[11] V.S. Guliyev, Y.Y. Mammadov, Function spaces and integral operators for the Dunkl operator on the real line, Khazar J. of Math., 2, No 4 (2006), 17-42.

[12] V.S.Guliyev, Y.Y.Mammadov, On fractional maximal function and fractional integrals associated with the Dunkl operator on the real line, $J$. Math. Anal. Appl., 353, No 1 (2009), 449-459.

[13] E.V. Guliyev, A. Eroglu, Y.Y. Mammadov, Necessary and sufficient conditions for the boundedness of Dunkl-Type fractional maximal operator in the Dunkl-type Morrey spaces, Abstract and Appl. Anal., 2010 (2010), Art. ID 976493, 10 pp.; doi:10.1155/2010/976493.

[14] J.J. Hasanov, A.M. Musayev, Oscillatory integral operators and their commutators in modified weighted Morrey spaces with variable exponent, Int. J. Appl. Math., 32, No 3 (2019), 521-535; doi:10.12732/ijam.v32i3.12.

[15] G. Hu, X. Shi, and Q. Zhang, Weighted norm inequalities for the maximal singular integral operators on spaces of homogeneous type, J. Math. Anal. Appl., 336, No 1 (2007), 1-117.

[16] Y.Y. Mammadov, On maximal operator associated with the Dunkl operator on R, Khazar J. of Math., 2, No 4 (2006), 59-70.

[17] Y.Y. Mammadov, S.A.Hasanli, On the boundedness of commutators Dunkl-type maximal operator in the Dunkl-type Morrey spaces, Caspian J. of Appl. Mathematics, Ecology and Economics, 6, No 1 (2018), 37-52.

[18] Y.Y. Mammadov, S.A. Hasanli, On the boundedness of Dunkl-type fractional integral operator in the generalized Dunkl-type Morrey spaces, Int. J. Appl. Math., 31, No 2 (2018), 211-230; doi:10.12732/ijam.v31i2.4.

[19] C.B. Morrey, On the solutions of quasi-linear elliptic partial differential equations, Trans. Amer. Math. Soc., 43 (1938), 126-166.

[20] M. Sifi, F. Soltani, Generalized Fock spaces and Weyl relations for the Dunkl kernel on the real line, J. Math. Anal. Appl., 270 (2002), 92-106.

[21] F. Soltani, $L_{p}$-Fourier multipliers for the Dunkl operator on the real line, J. Funct. Anal., 209 (2004), 16-35.

[22] F. Soltani, Littlewood-Paley operators associated with the Dunkl operator on R, J. Funct. Anal., 221 (2005), 205-225. 
[23] E.M. Stein, Harmonic Analysis: Real-Variable Methods, Orthogonality and Oscillatory Integrals, Princeton Univ. Press, Princeton, NJ (1993).

[24] M. Rösler, Bessel-type signed hypergroups on $R$, In: Probability Measures on Groups and Related Structures, XI Oberwolfach, 1994 (Eds. H. Heyer and A. Mukherjea), World Scientific, River Edge, NJ (1995), 292-304.

[25] M.E. Taylor, Analysis on Morrey spaces and applications to Navier-Stokes and other evolution equations, Comm. Partial Differential Equations, 17 (1992), 1407-1456.

[26] K. Trimeche, Paley-Wiener theorems for the Dunkl transform and Dunkl translation operators, Integr. Trans. Spec. Funct., 13 (2002), 17-38.

[27] G.N. Watson, A Treatise on Theory of Bessel Functions, Cambridge University Press, Cambridge (1966). 
